\title{
Antipsychotics in dementia: prevalence and quality of antipsychotic drug prescribing in UK mental health services
}

\author{
Thomas R. E. Barnes, Sube Banerjee, Noel Collins, Adrian Treloar, Samantha M. McIntyre \\ and Carol Paton
}

\section{Background}

Up to a quarter of people in the UK with a diagnosis of dementia are prescribed an antipsychotic in any year. The potential risks of such treatment are becoming clearer, but the benefits remain uncertain. Concern about the frequency and quality of such prescribing was expressed in the National Dementia Strategy for England in 2009.

\section{Aims \\ To provide an estimate of the prevalence of antipsychotic use for dementia in secondary mental health services in the UK and to collect data relevant to quality improvement initiatives for such prescribing practice.}

\section{Method}

In the context of a UK quality improvement programme, relevant clinical audit data were collected for patients with dementia under the care of specialist older people's mental health services.

\section{Results}

Fifty-four mental health National Health Service (NHS) trusts submitted data on 10199 patients. Of those patients without comorbid psychotic illness, 1620 (16\%) were prescribed an antipsychotic; the common clinical indications for such medication were agitation, psychotic symptoms, aggression and distress. Multivariable regression found younger age, care home or in-patient setting, vascular or Parkinson's disease dementia and greater severity of dementia to be all significantly associated with being prescribed antipsychotic medication. Of the 1001 (62\%) patients prescribed treatment for more than 6 months, only three-quarters had a documented review of therapeutic response in the previous 6 months.

\section{Conclusions}

The data reveal areas of relatively good current practice, including consideration of alternatives to antipsychotic medication and clear documentation of target symptoms. They also suggest areas for improvement, such as the frequency and quality of review of long-term medication. Strategies to reduce antipsychotic use should take account of the demographic and clinical variables predicting increased likelihood of antipsychotic prescription.

\section{Declaration of interest}

T.R.E.B. has received speaker funding from Lilly. A.T. has received research funding from Lundbeck. S.B. has received consultancy fees, speaker funding, research funding and educational support to attend conferences from pharmaceutical companies involved in the manufacture of antipsychotics, antidepressants and antidementia drugs, and has been employed by the Department of Health for England.
Around 800000 people in the UK have dementia. Approximately $80 \%$ will, during the course of the illness, exhibit non-cognitive symptoms and behaviour such as agitation, aggression, psychosis, wandering and sleep disturbance; these are referred to as behavioural and psychological symptoms of dementia (BPSD). ${ }^{1,2}$ The prevalence of agitation and aggression in those with moderate to severe dementia living in nursing homes has been reported to be over $50 \% .^{3}$ For the person with dementia, BPSD may be a way of communicating distress, whereas for carers it may be a cause of distress and a precipitant of transfer to institutional care. ${ }^{4}$ Therefore, BPSD are a legitimate target for treatment, with the aim of improving the quality of life for both people with dementia and their carers. Underlying causes of BPSD include psychotic experiences, and discomfort and pain due to physical illness such as infection, heart failure or arthritis. These symptoms may also reflect basic needs not being met, for example being hungry, thirsty, left in wet clothing or simply missing human contact. The treatment strategy requires the underlying cause to be identified, but where this proves difficult or is inadequately explored, custom and practice may be to use antipsychotic medication. ${ }^{5,6}$

The association between treatment with antipsychotic drugs and increased morbidity and mortality in people with dementia is clear. ${ }^{7}$ The older, first-generation antipsychotics were particularly associated with movement disorders. Studies of the newer, second-generation antipsychotics revealed an association with stroke; ${ }^{8}$ initially the safety warnings were specific to risperidone and olanzapine but as trials with other drugs were re-analysed and database studies undertaken it became apparent that it is likely that all antipsychotic drugs are implicated. ${ }^{9,10}$ In the UK the National Institute for Health and Clinical Excellence (NICE) clinical guideline for the management of dementia, published in 2006, recommended that antipsychotic drugs should not be prescribed for patients whose non-cognitive symptoms were of mild to moderate severity because of the increased risk of cerebrovascular adverse effects. ${ }^{11}$ This guideline stated that where such symptoms - specifically psychosis and/or agitated behaviour - are causing distress and are severe, an antipsychotic drug may be considered after target symptoms have been quantified and documented, comorbid conditions such as depression treated, non-pharmacological approaches tried, cardiovascular risk factors assessed, and a discussion held with the person with dementia and/or carers about the potential adverse effects of such treatment. Further, the prescription should be time-limited and regularly reviewed.

In 2009 the Department of Health in the UK commissioned an independent inquiry into the use of antipsychotics in people with dementia. ${ }^{6}$ The report concluded that up to a quarter of people 
with dementia in the UK are prescribed an antipsychotic at any one time, of whom only $20 \%$ would be likely to derive benefit, whereas $1 \%$ who would not otherwise have died would do so directly owing to the adverse effects of the medication. It also concluded that it should be possible to decrease the prevalence of use of antipsychotics by two-thirds within 36 months; that is, by October 2012. One recommendation was that there should be a cycle of audit to quantify the prevalence of antipsychotic use and improve the quality of decision-making around treatment initiation, and the frequency and quality of clinical review.

\section{Method}

The Prescribing Observatory for Mental Health (POMH-UK) facilitates audit-based, quality improvement programmes (QIPs) in mental health services in the UK. It is part of the College Centre for Quality Improvement (CCQI) at the Royal College of Psychiatrists. The POMH-UK invited all specialist mental health services in the UK to participate in a QIP focusing on the prevalence and quality of antipsychotic prescribing in people with dementia. The data collected in a baseline audit related to recommended prescribing practice in the NICE guideline on supporting people with dementia and their carers. ${ }^{11}$ Services were asked to submit data either for every eligible patient on a clinical team's caseload, or if this were not possible, to select every $n$th case in a way that maximised the chance of the sample being random and therefore representative of clinical practice. For each patient with dementia the following data were collected: age, gender, ethnicity, care setting, subtype and severity of dementia, other psychiatric diagnoses and psychotropic medicines prescribed. Where an antipsychotic drug was prescribed, the data collected included clinical indication, dosage and duration of treatment. For patients who had been prescribed an antipsychotic for less than 3 months, data were collected related to the underlying cause of BPSD and non-pharmacological treatment strategies. Where antipsychotic drug treatment had begun more than 3 months earlier, data relevant to medication review were collected.

\section{Statistical analysis}

Data were entered online using SNAP survey software, version 9 (www.snapsurveys.com/software) and analysed using SPSS version 15 on Windows XP. Simple descriptive statistics were used to determine the prevalence of antipsychotic use and the quality of treatment initiation and review. Chi-squared tests were used to compare the prescription of additional medication across sample subgroups. Binary logistic regression was used to explore whether potential predictive variables were associated with antipsychotic prescription. The effect of each variable was examined initially using a univariable analysis. Subsequently the joint effect of explanatory variables was examined in a multivariable analysis, using a backwards selection procedure to retain the statistically significant variables. This analysis was conducted for all patients in the sample prescribed one or more antipsychotic drugs, and then repeated excluding all those who had a diagnosis of a comorbid psychotic illness (schizophrenia spectrum disorder, bipolar disorder or psychotic depression). Where particular patient characteristics were unknown or not specified, the values were treated as missing.

\section{Results}

In March 2011 a total of 54 specialist mental health National Health Service (NHS) trusts participated in the QIP baseline audit, submitting data on 10199 patients with a diagnosis of dementia from 447 clinical teams. The demographic and clinical characteristics of the sample are shown in Table 1. Of this sample $1902(19 \%)$ patients were prescribed an antipsychotic; this figure falls to $1620(16 \%)$ if the focus is antipsychotic drug treatment of BPSD - that is, if patients with a comorbid psychotic illness are excluded. For the subsample of 1620 persons with no comorbid psychotic disorder (the BPSD group), the most commonly prescribed antipsychotics were quetiapine $(36 \%)$, risperidone (26\%), haloperidol (10\%), olanzapine (9\%) and amisulpride (9\%). For each of these drugs the median daily dosage was at the lower end of the range for their licensed indications. The target symptoms and clinical indications for such treatment were documented in the clinical records in $97 \%$ of cases; $27 \%$ had one indication documented, $20 \%$ had two, $20 \%$ had three and $30 \%$ had four or more. Table 2 shows the proportion of the BPSD group prescribed antipsychotic medication for each indication, and Table 3 provides details of other psychotropic medication prescribed to at least $3 \%$ of this group.

For the 417 patients who had begun treatment in the preceding 3 months and who did not have a comorbid psychotic disorder, there was documented evidence that before an antipsychotic had been prescribed a potential underlying cause of BPSD had been considered in $80 \%$ and that non-pharmacological interventions had been tried in $60 \%$.

\section{Factors associated with prescription of antipsychotic medication}

When potentially relevant clinical variables were examined individually in the total sample the following were found to be significantly associated $(P<0.001)$ with the prescription of antipsychotic medication: age, gender, care setting, whether or not detained under the Mental Health Act 1983, type and severity of dementia and a diagnosis of a comorbid psychotic illness. The results of the multivariable analysis indicated that age, patient setting, and type and severity of dementia were significantly $(P<0.001)$ associated with being prescribed an antipsychotic, but after adjusting for these variables there was no longer any significant effect of either gender or being detained under the Mental Health Act on the outcome. Thus, patients with a diagnosis of Alzheimer's disease and those whose dementia was mild in severity were least likely to be prescribed an antipsychotic. Also, older patients were less likely to be prescribed antipsychotics; patients aged 91 years or older had half the odds of being prescribed antipsychotics compared with those aged 70 years or younger. In terms of clinical setting, the greatest likelihood of being prescribed an antipsychotic was for in-patients on a psychiatric ward, or for those receiving care in a nursing home or in a private continuing care service, whereas the lowest likelihood was for community patients living in their own home.

The multivariable regression was repeated, excluding from the sample patients with a diagnosis of a comorbid psychotic disorder (Table 4). In this BPSD group, age, care setting, type of dementia and severity of dementia were all still found to be significantly associated with being prescribed an antipsychotic. The direction of the effects of each of these variables is similar to that observed in the initial univariable analyses of the total sample.

\section{Review of antipsychotic medication}

Of the BPSD group, $619(38 \%)$ had been prescribed an antipsychotic for up to 6 months, $326(20 \%)$ for $6-12$ months and $675(42 \%)$ for more than a year. Of the 1001 patients prescribed an antipsychotic for at least 6 months, 748 (75\%) 


\begin{tabular}{|c|c|c|c|}
\hline & \multirow[b]{2}{*}{$\begin{array}{l}\text { Total sample } \\
(n=10 \text { 199) }\end{array}$} & \multicolumn{2}{|c|}{ Antipsychotic prescribed $(n=1902)$} \\
\hline & & $\begin{array}{l}\text { Comorbid psychotic illness } \\
\qquad(n=282)\end{array}$ & $\begin{array}{l}\text { No comorbid psychotic illness } \\
\qquad(n=1620)\end{array}$ \\
\hline \multicolumn{4}{|l|}{ Gender, $n$ (\%) } \\
\hline Male & 3877 (38) & 89 (32) & $706(44)$ \\
\hline Female & $6322(62)$ & $193(68)$ & $914(56)$ \\
\hline \multicolumn{4}{|l|}{ Ethnicity, $n(\%)$} \\
\hline Black/Black British & $220(2)$ & $18(6)$ & $37(2)$ \\
\hline Mixed or other & $147(1)$ & $8(3)$ & $25(2)$ \\
\hline White/White British & $9015(88)$ & $229(81)$ & $1433(88)$ \\
\hline Asian & $283(3)$ & $17(6)$ & $35(2)$ \\
\hline Not specified or unknown & $534(5)$ & $10(4)$ & $90(6)$ \\
\hline \multicolumn{4}{|l|}{ Age, years } \\
\hline Median & 82 & 78 & 80 \\
\hline Interquartile range & $76-87$ & 73-83 & $75-86$ \\
\hline Minimum-maximum & 29-104 & $46-100$ & $29-100$ \\
\hline \multicolumn{4}{|l|}{ Age, $n(\%)$} \\
\hline 65 years or under & $548(5)$ & $29(10)$ & $113(7)$ \\
\hline $66-75$ years & 1709 (17) & $75(27)$ & $331(20)$ \\
\hline $76-85$ years & $4717(46)$ & $124(44)$ & $741(46)$ \\
\hline 86 years or over & 3225 (32) & $54(19)$ & $435(27)$ \\
\hline \multicolumn{4}{|l|}{ Patient setting, $n$ (\%) } \\
\hline Private continuing care & 56 (1) & $7(2)$ & $22(1)$ \\
\hline Psychiatric ward & $871(9)$ & 54 (19) & $394(24)$ \\
\hline EMI nursing home & $536(5)$ & $20(7)$ & $218(13)$ \\
\hline NHS continuing care & $244(2)$ & 9 (3) & $66(4)$ \\
\hline Nursing home & 396 (4) & $14(5)$ & $98(6)$ \\
\hline EMI residential home & $520(5)$ & $15(5)$ & $120(7)$ \\
\hline Medical/surgical ward & $66(1)$ & $1(0)$ & $16(1)$ \\
\hline Residential home & $876(9)$ & $40(14)$ & $164(10)$ \\
\hline Other/unknown & $66(1)$ & $2(1)$ & $7(0)$ \\
\hline Patient's own home & $6568(64)$ & $120(43)$ & $515(32)$ \\
\hline \multicolumn{4}{|l|}{ Type of dementia (ICD-10 code), $n$ (\%) } \\
\hline Dementia in Alzheimer's disease (F00) & 4989 (49) & $92(33)$ & $678(42)$ \\
\hline Dementia in Alzheimer's disease, atypical or mixed type (F00.2) & $2021(20)$ & $40(14)$ & $241(15)$ \\
\hline Vascular dementia (F01) & $1613(16)$ & $74(26)$ & $372(23)$ \\
\hline Dementia in Parkinson's disease (F02.3) & $228(2)$ & $9(3)$ & $59(4)$ \\
\hline Dementia, other $(\mathrm{FO} 2)$ & 357 (4) & $14(5)$ & $120(7)$ \\
\hline Unspecified dementia (F03) & $617(6)$ & $31(11)$ & $109(7)$ \\
\hline Dementia subtype not yet determined & $460(5)$ & $22(8)$ & $52(3)$ \\
\hline \multicolumn{4}{|l|}{ Severity of dementia } \\
\hline Mild & $2392(23)$ & $73(26)$ & $144(9)$ \\
\hline Moderate & $4667(46)$ & $129(46)$ & $614(38)$ \\
\hline Severe & 1976 (19) & $33(12)$ & 633 (39) \\
\hline Not known/not documented & 1164 (11) & $47(17)$ & $229(14)$ \\
\hline
\end{tabular}

had a documented medication review in the previous 6 months addressing therapeutic response. The bulk of these reviews (90\%) had been conducted by secondary care services, with smaller proportions being conducted by both secondary and primary care $(6 \%)$ or only by primary care $(4 \%)$.

\section{Discussion}

Our audit of over 10000 patients with dementia under the care of old age psychiatrists is the largest of its type conducted thus far in the UK. Specialist older people's mental health services are charged specifically with managing the most complex of cases including those with severe and complicated BPSD. This might be expected to result in an increased use of antipsychotics, but equally, the availability of non-drug alternatives in such services might enable the use of such drugs to be minimised. We found that $16 \%$ of such patients were currently prescribed antipsychotic medication for BPSD. This is lower than the $25 \%$ overall population rate for prescription of these medication to people with dementia estimated by Banerjee. ${ }^{6}$

\section{Patterns of prescribing}

In accord with published research, ${ }^{12}$ analysis of our audit data revealed that age, care setting and type and severity of dementia were all independently linked to the prevalence of antipsychotic drug prescribing in dementia. The highest prevalence of antipsychotic prescribing was found on psychiatric wards and in private continuing care. The relatively high prevalence in care homes for the elderly mentally infirm (EMI), ranging from $26 \%$ in EMI residential homes to $44 \%$ in EMI nursing homes, is consistent with previously reported estimates of $20-50 \%$ in the UK. ${ }^{3,13,14}$ As a predictor variable, care home setting may be partly a proxy for clinical variables not collected in our audit, such as level of dependency and the severity and complexity of behavioural problems such as agitation or aggression. The latter has been previously observed to be associated with a higher likelihood of 
Table 2 Documented clinical indications and target symptoms for antipsychotic medication in the BPSD group $(n=1620)$

\begin{tabular}{|c|c|}
\hline Indication $^{a}$ & $\begin{array}{c}\text { BPSD group }{ }^{b} \\
n(\%)\end{array}$ \\
\hline Agitation & $821(51)$ \\
\hline Evident or assumed psychotic symptoms & $736(45)$ \\
\hline Physical aggression & $683(42)$ \\
\hline Verbal aggression & $680(42)$ \\
\hline Distress & $425(26)$ \\
\hline Resisting help with activities of daily living & $320(20)$ \\
\hline Disturbed sleep & $239(15)$ \\
\hline Wandering & $236(15)$ \\
\hline Fear/anxiety & $194(12)$ \\
\hline Disinhibited behaviour & $156(10)$ \\
\hline Depression/low mood & $130(8)$ \\
\hline Unclear/other & $126(8)$ \\
\hline \multicolumn{2}{|c|}{$\begin{array}{l}\text { BPSD, behavioural and psychological symptoms of dementia. } \\
\text { a. Indications are not mutually exclusive; i.e. for an individual patient an antipsychotic } \\
\text { drug might be prescribed for more than one indication, or more than one drug might } \\
\text { be prescribed to target the same indication. } \\
\text { b. The BPSD group is the subgroup of patients with dementia but no comorbid } \\
\text { psychotic illness who are receiving antipsychotic medication. }\end{array}$} \\
\hline
\end{tabular}

antipsychotic use. ${ }^{15}$ A US study attributed variation in antipsychotic use to a nursing-home level 'prescribing culture' that exists in some institutions, ${ }^{16}$ whereas a Canadian study found that physical setting characteristics, specifically absence of a clock or calendar and telephone, may be relevant. ${ }^{15}$ Further studies are needed to understand these associations more fully.

Antipsychotics were more often prescribed for non-Alzheimer's disease dementia, despite even poorer evidence for efficacy and a potentially greater risk of harm. The highest prevalence of antipsychotic prescribing was in the small subgroup of patients with dementia associated with Parkinson's disease. This is in line with the findings of a large US database study, which found a significantly higher use of antipsychotic medication in patients with both Parkinson's disease and dementia compared with those with other subtypes of dementia. ${ }^{17}$ In our sample $30 \%$ of the 228 people with Parkinson's disease dementia were prescribed an antipsychotic. Possible explanations are that psychosis may be more common in this subgroup, perhaps partly due to treatment with dopaminergic drugs. It is likely that this complexity would have led to an appropriate, selective referral of such patients to psychiatric services.

The observed patterns of antipsychotic usage were consistent with previous studies, with doses tending to be at the lower end of the licensed range. There was high usage of quetiapine, accounting for over a third (36\%) of all antipsychotic prescriptions, despite a lack of positive efficacy data in dementia. ${ }^{18,19}$ The next most commonly prescribed antipsychotic was risperidone $(26 \%)$, the only antipsychotic currently licensed (on a restrictive set of indications) for the short-term management of BPSD. There was low usage of olanzapine and rare usage of clozapine. The common use of quetiapine might be partly explained by its perceived favourable tolerability profile, particularly in relation to extrapyramidal side-effects, and that it was not specifically implicated in the early Medicines and Healthcare Products Regulatory Agency warning of the risk of stroke with antipsychotic drugs in dementia. ${ }^{8}$ The only firstgeneration antipsychotic commonly used was haloperidol, and this was usually prescribed on an 'as required' (p.r.n.) basis.

\section{Quality of antipsychotic prescribing}

Our audit data suggest that the prescribing of antipsychotic drugs by specialist old age psychiatric services in the UK for people with dementia in 2010-2011 is largely compliant with treatment recommendations, in that alternative interventions had been considered in the majority of cases before medication was prescribed, and when it was prescribed there was clear documentation of the target symptoms. The most common clinical indications were agitation, evident or assumed psychotic symptoms, aggression and distress. This observed adherence to standards in the UK may reflect the desired effect of NICE guidelines. It is also consistent with the American experience of the impact of the US Food and Drug Administration advisory on second-generation antipsychotic use among all individuals and those 65 years or older with dementia. ${ }^{20}$ However, our audit also revealed aspects of antipsychotic prescribing practice that

\begin{tabular}{|c|c|c|c|c|}
\hline & \multicolumn{3}{|c|}{ Patients regularly prescribed this medicine, $n(\%)$} & \multirow[b]{2}{*}{$\begin{array}{c}\text { Antipsychotic subgroup } v \text {. } \\
\text { no antipsychotic subgroup } \\
\chi^{2}, P\end{array}$} \\
\hline & $\begin{array}{l}\text { Patients with dementia } \\
\text { but no psychotic illness } \\
\qquad(n=9825)\end{array}$ & $\begin{array}{l}\text { Subgroup prescribed } \\
\text { an antipsychotic }{ }^{a} \\
\qquad(n=1620)\end{array}$ & $\begin{array}{l}\text { Subgroup not prescribed } \\
\text { an antipsychotic } \\
\qquad(n=8205)\end{array}$ & \\
\hline Analgesic & $1184(12)$ & $238(15)$ & $946(12)$ & $12.71, P=0.001$ \\
\hline Anticholinergic & $108(1)$ & $44(3)$ & $64(<1)$ & $46.644, P<0.001$ \\
\hline \multicolumn{5}{|l|}{ Antidepressant } \\
\hline SSRI & 1679 (17) & $353(22)$ & $1326(16)$ & $30.257, P<0.001$ \\
\hline Trazodone & $473(5)$ & $131(8)$ & $342(4)$ & $45.325, P<0.001$ \\
\hline Other & $1062(11)$ & $272(17)$ & $790(10)$ & $71.978, P<0.001$ \\
\hline Carbamazepine & $140(1)$ & $49(3)$ & $91(1)$ & $35.344, P<0.001$ \\
\hline Cholinesterase inhibitor & $4213(43)$ & $441(27)$ & $3772(46)$ & 194.181, $P<0.001$ \\
\hline \multicolumn{5}{|l|}{ Benzodiazepine } \\
\hline Lorazepam & $312(3)$ & $133(8)$ & $179(2)$ & 159.897, $P<0.001$ \\
\hline Other & $492(5)$ & $166(10)$ & $326(4)$ & $111.941, P<0.001$ \\
\hline Memantine & $419(4)$ & $113(7)$ & $306(4)$ & $34.912, P<0.001$ \\
\hline Valproate & $228(2)$ & $88(5)$ & $140(2)$ & $82.851, P<0.001$ \\
\hline Z-hypnotic & $473(5)$ & $196(12)$ & 277 (3) & $224.631, P<0.001$ \\
\hline
\end{tabular}




\begin{tabular}{|c|c|c|}
\hline & Odds ratio $(95 \% \mathrm{Cl})$ & $P$ \\
\hline \multicolumn{3}{|l|}{ Age group, years } \\
\hline$\leqslant 70$ & 1 & \\
\hline $71-80$ & $0.95(0.76-1.18)$ & \\
\hline $81-90$ & $0.71(0.57-0.88)$ & \\
\hline $91+$ & $0.58(0.43-0.77)$ & $<0.001$ \\
\hline \multicolumn{3}{|l|}{ Care setting } \\
\hline Own home & 1 & \\
\hline Residential home & $2.40(1.93-2.97)$ & \\
\hline EMI residential home & $2.65(2.06-3.39)$ & \\
\hline Nursing home & $3.26(2.44-4.35)$ & \\
\hline EMI nursing home & $4.81(3.82-6.05)$ & \\
\hline Psychiatric ward & $6.93(5.71-8.43)$ & \\
\hline NHS continuing care & $2.14(1.50-3.07)$ & \\
\hline Private continuing care & $3.90(1.71-8.86)$ & \\
\hline Medical/surgical ward & $2.93(1.51-5.68)$ & $<0.001$ \\
\hline \multicolumn{3}{|l|}{ Type of dementia } \\
\hline Alzheimer's disease & 1 & \\
\hline Alzheimer's disease/mixed & $0.84(0.71-1.01)$ & \\
\hline Vascular & $1.60(1.35-1.89)$ & \\
\hline Parkinson's disease & $2.34(1.64-3.34)$ & \\
\hline Other & $1.71(1.26-2.31)$ & \\
\hline Unspecified & $1.14(0.86-1.50)$ & $<0.001$ \\
\hline \multicolumn{3}{|l|}{ Severity of dementia } \\
\hline Mild & 1 & \\
\hline Moderate & $1.90(1.56-2.32)$ & \\
\hline Severe & $3.27(2.62-4.08)$ & $<0.001$ \\
\hline
\end{tabular}

were suboptimal - in particular, the low level of review in cases prescribed such drugs long-term. In the subsample of patients prescribed an antipsychotic drug, almost two-thirds had been receiving it for at least 6 months, which is consistent with findings reported by Ballard et al. ${ }^{21}$ We found that only three-quarters of these patients had had a documented medication review in the previous 6 months. This group might be particularly vulnerable to suboptimal monitoring for several reasons. Although antipsychotic treatment for dementia is most commonly initiated in secondary care, prescribing responsibility is commonly transferred to primary care subsequently, with the risk of undefined responsibility for continued medication review. This may be further compounded by poor communication between general practitioners and psychiatrists. ${ }^{22}$ It has also been observed that primary care can be less successful than specialist mental health teams at withdrawing antipsychotics. ${ }^{23}$

\section{Other drugs prescribed}

The patients in this sample received a range of other medications. These included analgesics, antidepressants, valproate, benzodiazepines and z-hypnotics; all were prescribed more often for the subgroup of patients who were prescribed an antipsychotic. It is possible that these drugs were also being used to target BPSD. Indeed, there is some evidence that pain is associated with behavioural disturbances and depression in people with dementia, and that analgesics may be as effective as antipsychotics in reducing agitation in such cases. ${ }^{24}$ There is also limited evidence to suggest that antidepressants may have a modest effect in reducing agitation, although not depression, in people with dementia. ${ }^{25,26}$ In contrast, there is no evidence supporting the efficacy of valproate but some indicating potential harm. ${ }^{27}$ Benzodiazepines have been tentatively recommended for the management of mild agitation, although there is no study of people with dementia. These drugs, alongside the z-hypnotics, are likely to be seen as more directly targeting agitation and insomnia, and as being safer in some ways than antipsychotics; but both groups of drugs are associated with an increased risk of falls.

Cholinesterase inhibitors, conversely, were less likely to be prescribed in the subsample of patients prescribed an antipsychotic. This may be due to the inverse relationship that exists between cognition and BPSD; cholinesterase inhibitors are indicated for mild to moderate dementia, whereas BPSD are more prevalent in moderate to severe dementia. Most antipsychotics also have anticholinergic activity, which is associated with hazards for older people such as urinary retention, blurred vision and impaired cognition. ${ }^{28}$ Further, this activity would be likely to negate the effects of the cholinesterase inhibitors in the $25 \%$ of patients prescribed both an antipsychotic and a cholinesterase inhibitor. Such co-prescribing has been seen in other large samples of people with dementia, ${ }^{29}$ although the efficacy and tolerability of such regimens have not been formally tested in randomised controlled trials.

\section{Limitations and strengths of the study}

The audit data were collected by local staff, so there may have been error and variability in the data abstraction process. Also, the local clinical services were responsible for selecting which clinical teams would participate, and the numbers and types of services involved varied by respondent. Although these factors might act to limit the generalisability of the data, this effect is probably minimal given the large sample size and the involvement of the large majority of English mental health NHS trusts. Thus, the findings may be seen as representative of current clinical practice in specialist mental health services, although they cannot be readily extrapolated to primary care.

\section{Implications for quality improvement}

The main aim of this audit was to provide performance data (benchmarked against clinical practice standards) to participating services, stimulating reflective practice, as part of a QIP. Further, our findings identify two priorities for quality initiatives in this area: first, the high prevalence of antipsychotic prescribing on psychiatric wards and in care homes, and second, the frequency and thoroughness of the review of medication for patients prescribed antipsychotics beyond the short term. Individual trusts participating in this audit have started to publish and use their data to benchmark and identify priorities for improvement. Other local audits are reported to have led to improvements, including a modest reduction in the prescribing of antipsychotic drugs in nursing homes following the introduction of a checklist and 6-monthly reviews. ${ }^{30}$ Audit now appears embedded in secondary care as a quality improvement mechanism. However, other techniques have been reported to improve prescribing practice in vulnerable groups of patients. These include clearer communication of information around prescribing and followup; formal integration of a clinical pharmacist into community healthcare systems; and incentivised practice such as the Quality and Outcomes Framework. ${ }^{31,32}$

\section{Implications for future practice}

The findings we report here are important for two main reasons. First, they indicate that mental health teams dealing with patients with dementia and a high level of BPSD are able to manage all but $16 \%$ without recourse to antipsychotics. However, this is an overall prevalence figure for mental health services, and our data show that the proportion of patients prescribed antipsychotics 
varies markedly, depending on clinical setting and on particular demographic and clinical variables, such as being on a psychiatric ward, living in a care home, or having more severe dementia or dementia related to Parkinson's disease. Initiatives to change practice should take account of these factors, at the same time acknowledging that the optimal use of antipsychotic medication in such clinical subgroups remains unknown. Second, the benchmarked data generated from the audit allow the clinical services that participated to reflect on their own practice in relation to antipsychotic prescribing in patients with a diagnosis of dementia. The data indicate areas of relatively good practice, such as consideration of alternative interventions, and clear documentation of target symptoms when the medication is initiated. They also reveal areas for improvement, such as the frequency and quality of the review of long-term prescription of antipsychotic medication. Any impact on prescribing practice will be assessed in the QIP re-audit and in potential supplementary audits in the next few years.

Thomas R. E. Barnes, DSc, Imperial College London, and the Prescribing Observatory for Mental Health (POMH), Royal College of Psychiatrists' Centre for Quality Improvement, London; Sube Banerjee, MD, King's College London, Institute of Psychiatry; Noel Collins, MB BS, Surrey and Borders Partnership National Health Service (NHS) Foundation Trust, Leatherhead, Surrey; Adrian Treloar, MRCPsych, Oxleas NHS Foundation Trust, Dartford, Kent; Samantha M. McIntyre, BA, POMH, Royal College of Psychiatrists' Centre for Quality Improvement, London; Carol Paton, BSc, Imperial College London, and POMH, Royal College of Psychiatrists' Centre for Quality Improvement, London, UK

Correspondence: Thomas R. E. Barnes, Centre for Mental Health, Faculty of Medicine, Imperial College, Claybrook Centre, 37 Claybrook Road, London W6 8LN, UK. Email: t.r.barnes@imperial.ac.uk

First received 14 Dec 2011, final revision 11 Apr 2012, accepted 30 Apr 2012

\section{Funding}

The UK Prescribing Observatory for Mental Health is funded by the subscriptions of member healthcare organisations.

\section{Acknowledgements}

Acknowledgements are due to the participating mental health National Health Service (NHS) trusts and the NHS clinicians and administrators who collected the audit data. Thanks are due to Paul Bassett for statistical advice, Mary Murrell for expert advice and members of the Prescribing Observatory for Mental Health (POMH-UK) team, Krysia Zalewska and Sumera Bhatti, for administrative support. The POMH-UK is based at the Centre for Quality Improvement at the Royal College of Psychiatrists' Research Unit (www.rcpsych.ac.uk/pomh).

\section{References}

1 Finkel SI, Costa e Silva J, Cohen G, Miller S, Sartorius N. Behavioral and psychological signs and symptoms of dementia: a consensus statement on current knowledge and implications for research and treatment. Int Psychogeriatr 1996; 8 (suppl 3): 497-500.

2 Parnetti L, Amici S, Lanari A, Gallai V. Pharmacological treatment of noncognitive disturbances in dementia disorders. Mech Ageing Dev 2001; 122 2063-9.

3 Testad I, Auer S, Mittelman M, Ballard C, Fossey J, Donabauer Y, et al. Nursing home structure and association with agitation and use of psychotropic drugs in nursing home residents in three countries: Norway, Austria and England. Int J Geriatr Psychiatry 2010; 25: 725-31.

4 Coehlo DP, Hooker K, Bowman S. Institutional placement of persons with dementia: what predicts occurrence and timing? J Fam Nurs 2007; 13: 253-77.

5 Burns A, lliffe S. Dementia. BMJ 2009; 338: b75.

6 Banerjee S. The Use of Antipsychotic Medication for People With Dementia: Time for Action. Department of Health, 2009.

7 Kales HC, Kim HM, Zivin K, Valenstein M, Seyfried LS, Chiang C, et al. Risk of mortality among individual antipsychotics in patients with dementia. Am J Psychiatry 2012; 169: 71-9.

8 Medicines and Healthcare Products Regulatory Agency. New Advice Issued on Risperidone and Olanzapine. MHRA, 2004 (http:// www.mhra.gov.uk/Safetyinformation/Safetywarningsalertsandrecalls/ Safetywarningsandmessagesformedicines/CON002047).
9 Schneider LS, Dagerman KS, Insel P. Risk of death with atypical antipsychotic drug treatment for dementia: meta-analysis of randomized placebocontrolled trials. JAMA 2005; 294: 1934-43.

10 Prince MJ, Costa E. A Systematic Review of the Evidence on the Efficacy and Harm Associated with the Use of Conventional and Atypical Antipsychotics for the Treatment of Behavioural Disturbance and Psychosis in Dementia. Department of Health, 2009.

11 National Institute for Health and Clinical Excellence. Dementia. Guideline on Supporting People With Dementia and Their Carers in Health and Social Care. (Clinical Guideline CG42). NICE, 2006 (http://www.nice.org.uk/nicemedia/live/ 10998/30318/30318.pdf)

12 Alanen HM, Finne-Soveri H, Noro A, Leinonen E. Use of antipsychotics in older home care patients in Finland. Drugs Aging 2008; 25: 335-42.

13 McGrath AM, Jackson GA. Survey of neuroleptic prescribing in residents of nursing homes in Glasgow. BMJ 1996; 312: 611-2.

14 Alldred DP, Petty DR, Bowie P, Zermansky AG, Raynor DK. Antipsychotic prescribing patterns in care homes and relationship with dementia. Psychiatr Bull 2007; 31: 329-32.

15 Monette J, Alessa W, McCusker, J, Cole M, Voyer P, Champoux N, et al. Association of resident and room characteristics with antipsychotic use in long-term care facilities (LTCF). Arch Gerontol Geriatr 2012; 55: 66-9.

16 Chen Y, Briesacher BA, Field TS, Tjia J, Lau DT, Gurwitz JH. Unexplained variation across US nursing homes in antipsychotic prescribing rates. Arch Intern Med 2010; 170: 89-95.

17 Weintraub D, Chen $\mathrm{P}$, Ignacio RV, Mamikonyan E, Kales HC. Patterns and trends in antipsychotic prescribing for Parkinson disease psychosis. Arch Neurol 2011; 68: 899-904.

18 Tariot PN, Schneider L, Katz IR, Mintzer JE, Street J, Copenhaver M, et al. Quetiapine treatment of psychosis associated with dementia: a double-blind, randomized, placebo-controlled clinical trial. Am J Geriatr Psychiatry 2006; 14: $767-76$.

19 Sultzer DL, Davis SM, Tariot PN, Dagerman KS, Lebowitz BD, Lyketsos CG, et al. Clinical symptom responses to atypical antipsychotic medications in Alzheimer's disease: phase 1 outcomes from the CATIE-AD effectiveness trial. Am J Psychiatry 2008; 165: 844-54.

20 Dorsey ER, Rabbani A, Gallagher SA, Conti RM, Alexander GC. Impact of FDA black box advisory on antipsychotic medication use. Arch Intern Med 2010; 170: 96-103.

21 Ballard C, Hanney ML, Theodoulou M, Douglas S, McShane R, Kossakowski K, et al. The dementia antipsychotic withdrawal trial (DART-AD): long-term follow-up of a randomised placebo-controlled trial. Lancet Neurol 2009; 8: $151-7$.

22 Soyinka A, Lawley D. Antipsychotic prescribing for behavioural and psychological symptoms of dementia. Psychiatr Bull 2007; 31: 176-8.

23 Malone $D$, Bradley $P$, Lindesay J. Olanzapine and risperidone prescriptions for people with dementia in care. Psychiatr Bull 2007; 31: 459-62.

24 Husebo BS, Ballard C, Sandvik R, Nilsen OB, Aarsland D. Efficacy of treating pain to reduce behavioural disturbances in residents of nursing homes with dementia: cluster randomised clinical trial. BMJ 2011; 343: d4065

25 Seitz DP, Adunuri N, Gill SS, Gruneir A, Herrmann N, Rochon P. Antidepressants for agitation and psychosis in dementia. Cochrane Database Syst Rev 2011; 2: CD008191.

26 Banerjee S, Hellier J, Dewey M, Romeo R, Ballard C, Baldwin R, et al. Sertraline or mirtazapine for depression in dementia (HTA-SADD): a randomised, multicentre, double-blind, placebo-controlled trial. Lancet 2011; 378: 403-11.

27 Lonergan $\mathrm{E}$, Luxenberg J. Valproate preparations for agitation in dementia. Cochrane Database Syst Rev 2009; 3: CD003945.

28 Fox C, Richardson K, Maidment ID, Savva GM, Matthews FE, Smithard D, et al. Anticholinergic medication use and cognitive impairment in the older population: the medical research council cognitive function and ageing study. J AM Geriatr Soc 2011; 59: 1477-83.

29 Langballe EM, Engdahl B, Seabaek G, Nordeng H. Concomitant use of antidementia drugs with psychotropic drugs in Norway - a population-based study. Pharmacoepidemiol Drug Saf 2011; 20: 1319-26.

30 Morrison A. Antipsychotic prescribing in nursing homes: an audit report Qual Primary Care 2009; 17: 359-62.

31 Schembri Wismayer F, Sipos A. Neuroleptics in dementia: two consecutive surveys of prescribing practice at the interface of hospital and primary care. Psychiatr Bull 1999; 23: 409-12.

32 Stuijt CC, Franssen EJ, Egberts AC, Hudson SA. Appropriateness of prescribing among elderly patients in a Dutch residential home: observational study of outcomes after a pharmacist-led medication review. Drugs Aging 2008; 25: 947-54. 